\title{
LARGE DEVIATIONS FOR IDEAL QUANTUM SYSTEMS
}

\author{
Joel L. Lebowitz ${ }^{1,2, *} \quad$ Marco Lenci ${ }^{1, \dagger}$ \\ Herbert Spohn $3, \ddagger$ \\ ${ }^{1}$ Department of Mathematics and ${ }^{2}$ Department of Physics \\ Rutgers University \\ Piscataway, NJ 08854, U.S.A. \\ ${ }^{3}$ Zentrum Mathematik and Physik Department \\ Technische Universität \\ 80290 München, Germany \\ Dedicated to Jim McGuire \\ ON THE OCCASION OF HIS 65TH BIRTHDAY \\ June 1999
}

\begin{abstract}
We consider a general $d$-dimensional quantum system of non-interacting particles, with suitable statistics, in a very large (formally infinite) container. We prove that, in equilibrium, the fluctuations in the density of particles in a subdomain $\Lambda$ of the container are described by a large deviation function related to the pressure of the system. That
\end{abstract}

\footnotetext{
${ }^{*}$ E-mail: lebowitz@math.rutgers.edu

${ }^{\dagger}$ E-mail: lenci@math.rutgers .edu

${ }^{\ddagger}$ E-mail: spohn@mathematik.tu-muenchen. de
} 
is, untypical densities occur with a probability exponentially small in the volume of $\Lambda$, with the coefficient in the exponent given by the appropriate thermodynamic potential. Furthermore, small fluctuations satisfy the central limit theorem.

\section{Introduction}

Statistical mechanics is the bridge between the microscopic world of atoms and the macroscopic world of bulk matter. In particular it provides a prescription for obtaining macroscopic properties of systems in thermal equilibrium from a knowledge of the microscopic Hamiltonian. This prescription becomes mathematically precise and elegant in the limit in which the size of the system becomes very large on the microscopic scale (but not large enough for gravitational interactions between the particles to be relevant). Formally this corresponds to considering neutral or charged particles with effective translation invariant interactions inside a container and taking the infinite-volume or thermodynamic limit (TL). This is the limit in which the volume $|V|$ of the container $V$ grows to infinity along some specified regular sequences of domains, say cubes or balls, while the particle and energy density approach some finite limiting value $[\mathbb{R}, \mathbb{F}, \mathbf{G}, \mathbb{T}]$. This limit provides a precise way for eliminating "finite size" effects.

It is then an important result (a theorem, under suitable assumptions) of rigorous statistical mechanics that the bulk properties of a physical system, computed from the thermodynamic potentials via any of the commonly used Gibbs ensembles (microcanonical, canonical, grand canonical, etc.), have well defined "equivalent" TL's [R, F, G]. These free energy densities are furthermore proven to be the same for a suitable class of "boundary conditions" (b.c.), describing the interaction of the system with the walls and the "outside" of its container. When this independence of b.c. is "strong enough", the bulk free energies also yield information about normal fluctuations, as well as large deviations, in particle number and energy, inside large regions of macroscopic systems. The theory of such fluctuations is at the present time well developed for classical systems [R, G, vFS], but almost nonexistent for quantum ones. It is the purpose of this note to make a beginning towards such a quantum theory. This is clearly desirable since the real world is quantum mechanical, with the classical description being an essentially uncontrolled approximation, albeit a very good one in many circumstances. 


\subsection{Classical systems}

We begin by considering a classical system of $N$ particles of mass $m$ in a domain, say a cubical box $V \subset \mathbb{R}^{d}$, interacting with each other through a sufficiently rapidly decaying pair potential $\phi(r)$, e.g., a Lennard-Jones potential. The Hamiltonian of the system is then given by

$$
H(N, V ; b)=\frac{1}{2 m} \sum_{i=1}^{N} \mathbf{p}_{i}^{2}+\frac{1}{2} \sum_{1 \leq i \neq j \leq N} \phi\left(r_{i j}\right)+\sum_{i=1}^{N} u_{b}\left(\mathbf{r}_{i}\right),
$$

where $\mathbf{p}_{i} \in \mathbb{R}^{d}, \mathbf{r}_{i} \in V, r_{i j}=\left|\mathbf{r}_{i}-\mathbf{r}_{j}\right|$, and $u_{b}\left(\mathbf{r}_{i}\right)$ represents the interaction of the $i$-th particle with the world outside of the boundary of $V$. This boundary interaction (indicated and in the sequel by $b$ ) is in addition to the action of the implicitly assumed "hard wall" which keeps the particles confined to $V$. The dynamic effect of the latter is to reflect the normal component of the particle's momentum when it hits the wall. However, sometimes it is convenient to replace it with periodic boundary conditions [FW].

For a macroscopic system in equilibrium at reciprocal temperature $\beta$ and chemical potential $\mu$, the grand canonical Gibbs ensemble then gives the probability density for finding exactly $N$ particles inside $V \subset \mathbb{R}^{d}$ at the phase point $X_{N}=\left(\mathbf{r}_{1}, \mathbf{p}_{1}, \ldots, \mathbf{r}_{N}, \mathbf{p}_{N}\right)=\left(\mathbf{R}_{N}, \mathbf{P}_{N}\right) \in \Gamma_{N}=V^{N} \times \mathbb{R}^{d N}$ as

$$
\nu\left(X_{N} \mid \beta, \mu, V, b\right)=\frac{(N !)^{-1} h^{-N d} \exp [-\beta(H(N, V ; b)-\mu N)]}{\Xi(\beta, \mu \mid V, b)},
$$

where $\Xi$ is the grand canonical partition function

$$
\begin{aligned}
\Xi & =\sum_{N=0}^{\infty}(N !)^{-1} \lambda_{B}^{-d N} e^{\beta \mu N} \int_{V^{N}} d \mathbf{r}_{1} \cdots d \mathbf{r}_{N} e^{-\beta / 2 \sum \phi\left(r_{i j}\right)-\beta \sum u_{b}\left(\mathbf{r}_{i}\right)}= \\
& =\sum_{N=0}^{\infty} e^{\beta \mu N} Q(\beta, N \mid V, b),
\end{aligned}
$$

where $Q(\beta, N \mid V, b)$ is the canonical partition function. We use $h^{d N}, h$ being Planck's constant, as the unit of volume in the phase space $\Gamma_{N}$, so $\lambda_{B}=$ $h \sqrt{\beta /(2 \pi m)}$ is the de Broglie wave length. The finite-volume, boundary condition dependent, grand canonical pressure is

$$
p(\beta, \mu \mid V, b)=\beta^{-1}|V|^{-1} \log \Xi(\beta, \mu \mid V, b) .
$$


Taking now the TL, $V \nearrow \mathbb{R}^{d}$, we obtain, for a suitable class of b.c., an instrinsic (b.c. independent) grand canonical pressure $p(\beta, \mu)$. This is related to the Helmholtz free energy density $a(\beta, \rho)$ obtained from the TL of the canonical ensemble, i.e., $\Xi$ is replaced by $Q^{-1}$ in (1.4) and the limit is taken in such a way that $N /|V| \rightarrow \rho$, a specified particle density. The relation between $p$ and $a$ is given by the usual thermodynamic formula involving the Legendre transform

$$
p(\beta, \mu)=\sup _{\rho}[\rho \mu-a(\beta, \rho)]=\pi(\beta, \bar{\rho}),
$$

where $\pi(\beta, \rho)$ is the TL of the canonical pressure

$$
\pi(\beta, \rho)=-\rho^{2} \frac{\partial(a / \rho)}{\partial \rho}
$$

and

$$
\bar{\rho}(\beta, \mu)=\frac{\partial p}{\partial \mu}(\beta, \mu)
$$

is the average density in the grand canonical ensemble.

At a first order phase transition $p(\beta, \mu)$ is discontinuous and the left/right limits of the derivative on the r.h.s. of (1.7) give the density in the coexisting phases. In our discussion we shall resctrict ourselves to values of the parameters $\beta$ and $\mu$ where the system is in a unique phase. We can of course also go from the grand canonical pressure to the Helmholtz free energy density by the inverse of (1.5),

$$
-a(\beta, \rho)=\sup _{\mu}[p(\beta, \mu)-\rho \mu]
$$

Let $P\left(N_{V} \in \Delta|V| \mid \beta, \mu, V, b\right)$ be the probability of finding a particle density in $V$ which lies in the interval $\Delta=\left[n_{1}, n_{2}\right]$, i.e., between the densities $n_{1}$ and $n_{2}$. Then, for $b$ in the right class of b.c., we have (almost by definition) that

$$
\lim _{V \nearrow \mathbb{R}^{d}}|V|^{-1} \log P\left(N_{V} \in \Delta|V| \mid \beta, \mu, V, b\right)=-\inf _{n \in \Delta}[a(\beta, n)-a(\beta, \bar{\rho})],
$$

where $\bar{\rho}$ is given by (1.7). In probabilistic language, this means that $a(\beta, n)$ is the "large deviation functional" for density fluctuations. (Note that $a(\beta, \rho)$ may be infinite for some values of $\rho$, i.e., when $\phi(r)=\infty$, for $r<D$, and $\rho$ is above the close-packing density of balls with diameter $D$ ). 
On the other hand, the fluctuations in all of $V$ are clearly ensemble dependent (they are nonexistent in the canonical ensemble) and therefore not so physical. More relevant are the fluctuations not in the whole volume $V$ but in a region $\Lambda$ inside $V$. Of particular interest is the case when $\Lambda$ is very large on the microscopic scale but still very small compared to $V$. The proper idealization of this situation is to first take the TL of $V \nearrow \mathbb{R}^{d}$ and then let $\Lambda$ itself become very large. We are thus interested in the probability $P\left(N_{\Lambda} \in \Delta|\Lambda| \mid \beta, \mu\right)$, for $\Lambda$ a large region in an infinite system obtained by taking the TL of $V$. This probability should now be an intrinsic property of a uniform single-phase macroscopic system characterized either by a chemical potential $\mu$ or by a density $\rho$.

A little thought shows that this probability corresponds to considering the grand canonical ensemble of a system of particles on a domain $\Lambda$ with boundary interactions of the type

$$
u_{b}\left(\mathbf{r}_{i}\right)=\sum_{k=1}^{\infty} \phi\left(\left|\mathbf{r}_{i}-\mathbf{x}_{k}\right|\right), \quad \mathbf{r}_{i} \in V, \mathbf{x}_{k} \in V^{c}
$$

i.e., we imagine that the boundary interactions come from particles of the same type as those inside $\Lambda$, specified to be at positions $\mathbf{x}_{1}, \mathbf{x}_{2}, \ldots$ outside $\Lambda$. These positions must then be averaged according to the infinite-volume Gibbs measure. It follows then, from the independence of the bulk properties of the system of the boundary conditions, that equation (1.9) is still correct, that is

$$
\lim _{\Lambda \nearrow \mathbb{R}^{d}}|\Lambda|^{-1} \log P\left(N_{\Lambda} \in \Delta|\Lambda| \mid \beta, \mu\right)=-\inf _{n \in \Delta}[a(\beta, n)-a(\beta, \rho)] .
$$

This relation is indeed a theorem for classical systems, under fairly general conditions [G, VFS, O].

\subsection{Quantum systems}

It is equation (1.11) and similar formulas for fluctuations in the energy density which we want to generalize to quantum systems. To do this, we begin by considering the boundary conditions imposed on the $N$-particle wave functions $\Psi\left(\mathbf{r}_{1}, \ldots, \mathbf{r}_{N} \mid V\right)$ for a quantum system in the domain $V$. Usually this is done by requiring that whenever any $\mathbf{r}_{i}$ is at the boundary of $V, \mathbf{r}_{i} \in \partial V$, 
then $\Psi$ is equal to $\alpha$ times its normal derivative

$$
\Psi\left(\mathbf{r}_{1}, \ldots, \mathbf{r}_{N} \mid V\right)=\alpha \mathbf{n}_{i} \cdot \frac{\partial}{\partial \mathbf{r}_{i}} \Psi\left(\mathbf{r}_{1}, \ldots, \mathbf{r}_{N} \mid V\right)
$$

with $\alpha=0$ corresponding to Dirichlet and $\alpha=+\infty$ to Neumann boundary conditions.

Denote by $b_{\alpha}$ the elastic boundary condition (1.12). The existence of the TL of the grand canonical $p\left(\beta, \mu \mid V, b_{0}\right)$ has been proven for quantum systems with stable potentials $[\mathbb{R}]$, and for positive potentials it is established that the pressure does not depend on $b_{\alpha}[\mathrm{Ro}]$. But, as far as we are aware, the dependence on $u_{b}\left(\mathbf{r}_{i}\right)$ has not been studied systematically, with the exception of the regime covered by the low-density expansion of Ginibre Gi, BR. This only shows that the dependence on the boundary is not so well understood for continuous quantum systems.

To investigate the density fluctuations in quantum systems we note that the momentum variables did not play any role in the derivation of (1.9) and (1.11) for classical systems. The only thing relevant, when considering particle number fluctuations, is the probability density in the configuration space. This is given for a classical system by integrating $\nu$ in (1.2) over the momentum variables, whose distribution is always a product of Gaussians (Maxwellians). For a quantum system, where the analog of (1.2) is the density matrix $\widehat{\nu}$, the configuration probability density is given by the diagonal elements of $\widehat{\nu}$ in the position representation. For the grand canonical ensemble this can be written as

$$
\widehat{W}\left(\mathbf{R}_{N} \mid \beta, \mu, V, b_{\alpha}\right)=\frac{e^{\beta \mu N} \sum_{\gamma}\left|\Psi_{\gamma}\left(\mathbf{R}_{N} \mid V, b_{\alpha}\right)\right|^{2} e^{-\beta E_{\gamma}}}{\widehat{\Xi}},
$$

where $\Psi_{\gamma}$ and $E_{\gamma}$ are the eigenstates and eigenvalues of $H_{N}$ with the suitable statistics and $b_{\alpha}$ b.c. $\mathbb{R}, \mathbb{B}$.

It is clear from the derivation of the TL $\mathbb{R}, \mathbb{E}]$ that, when $\phi(r)$ is superstable, the TL for the canonical ensemble exists for all $\rho \in\left[n_{1}, n_{2}\right]$ with b.c. $b_{\alpha}$. Then (1.9) carries over to quantum systems. The real problem is how to prove (1.11) for these systems. $\widehat{W}$ is no longer a Gibbs measure with a pair potential as interaction and there is no good reason to expect it to be a Gibbs measure for any other "reasonable" many-body potential vFS. (Even if the latter were the case, this potential would almost certainly depend on the density and temperature of the system and would therefore not carry 
directly any information on (1.11).) It might in fact appear that there is no strong reason why (1.11) should hold for quantum systems. The reason for expecting it to be true is that it is a thermodynamic type relation and such relations are in general unaffected by the transition from the classical to the quantum formalism. More explicitly, we see the difference between (1.9) and (1.11) as involving only boundary type quantities which should become irrelevant when $\Lambda$ is of macroscopic size. The proof of such a statement is however far from obvious (to us) and we therefore devote the rest of this note to proving it in the (technically) simplest case where there are no interactions between the particles, i.e., the ideal gas with either Bose-Einstein or FermiDirac statistics. It turns out that even in this case the proof requires a certain amount of work.

To finish this introduction, we note that the same reasoning which leads to (1.11) also gives the well known result that the variance of $N_{\Lambda}$, divided by $|\Lambda|$, is given, for $|\Lambda| \nearrow \infty$, by the compressibility $\left\langle\left(N_{\Lambda}-\rho|\Lambda|\right)^{2}\right\rangle /|\Lambda| \rightarrow$ $\beta^{-1}\left(\partial^{2} p / \partial \mu^{2}\right)(\beta, \mu)$. Furthermore, a central limit theorem for the random variable $\xi=\lim _{|\Lambda| \nearrow_{\infty}}\left(N_{\Lambda}-\rho|\Lambda|\right) / \sqrt{\rho|\Lambda|}$ holds. These results are also expected to remain valid for quantum systems and are proven here in the non-interacting case.

\section{Main results}

We consider a $d$-dimensional square box $V=[-\ell / 2, \ell / 2]^{d}$. For computational convenience we choose periodic boundary conditions. The infinite-volume thermal state we construct below does not depend on this particular choice of the boundary conditions, however [BR]. In $V$ there is an ideal fluid (either Fermi or Bose) in thermal equilibrium, as described by the grand canonical ensemble. We label the Bose fluid, shorthand BE, with the index + and the Fermi fluid, shorthand FD, with the index - and introduce the Fock space

$$
\mathcal{F}_{ \pm}^{V}=\mathbb{C} \oplus \bigoplus_{n=1}^{\infty} L_{ \pm}^{2}\left(V^{n}\right)
$$

where $L_{ \pm}^{2}\left(V^{n}\right)$ is the $n$-particle space of all symmetric, resp. antisymmetric, square-integrable functions on $V^{n}$. Of course, for $n=1, L_{ \pm}^{2}(V)=L^{2}(V)$. In the sequel, in order to keep the notation light, we will often drop sub- or superscripts whenever there is no ambiguity. 
Particles do not interact. Therefore the many-particle Hamiltonian in the box $V$ can be written conveniently in the form

$$
H_{V}=\bigoplus_{n=0}^{\infty} \sum_{i=1}^{n} \underbrace{1 \otimes \cdots \otimes h_{V} \otimes \cdots \otimes 1}_{i^{\text {th }} \text { position out of } n}
$$

where $h_{V}$, the one-particle Hamiltonian on $L^{2}(V)$, is defined through the one-particle energy $\epsilon(k)$ in momentum space. This means that, if $|k\rangle$ denotes the momentum eigenvector (represented in $L^{2}(V)$ as $\psi_{V}^{(k)}(x)=e^{i k \cdot x}$ ), then $h_{V}|k\rangle=\epsilon(k)|k\rangle$ with $k \in V^{\prime}=(2 \pi \mathbb{Z} / \ell)^{d}$, the dual of $V$.

We assume $\epsilon(k)$ to be continuous, $\epsilon(0)=0$ as a normalization, and $\epsilon(k)>$ 0 for $k \neq 0$. Also $\epsilon(k) \approx|k|^{\gamma}$ for small $k$ and $\epsilon(k) \geq|k|^{\alpha}$ for large $k$, with $\alpha, \gamma>0$. Furthermore we require

$$
\int d^{d} x\left|\int d^{d} k e^{i k \cdot x} \frac{1}{e^{\beta \epsilon(k)-\beta \mu}-\varepsilon}\right|<\infty
$$

for $\varepsilon= \pm 1, \beta>0$, and suitable $\mu$.

The standard example of a non-relativistic, resp. relativistic, kinetic energy for a particle of mass $m$ is $\epsilon(k)=k^{2} /(2 m)$, resp. $\epsilon(k)=\sqrt{m^{2} c^{4}+k^{2} c^{2}}-$ $m c^{2}$ (having set Planck's constant $\hbar=1$ ). Both functions satisfy the above conditions. The relativistic case includes $m=0$, although this is not immediately obvious, cf. Appendix A.1 for details.

We observe that $H_{V}$ may be rewritten as a quadratic form in the creation and annihilation operators on the Fock space $\mathcal{F}$. Let $a_{k}^{*}$ be the operator that creates a particle in the state $|k\rangle$ and $a_{k}$ the corresponding annihilator. Then

$$
H_{V}=\sum_{k} \epsilon(k) a_{k}^{*} a_{k}=\sum_{j, k}\left\langle j\left|h_{V}\right| k\right\rangle a_{j}^{*} a_{k}=\left\langle\mathbf{a}\left|h_{V}\right| \mathbf{a}\right\rangle .
$$

We fix $\beta>0$ and $\mu \in \mathbb{R}$ for FD, resp., $\mu<0$ for BE. The grand canonical state in the volume $V$ is defined by

$$
\langle A\rangle_{ \pm, \mu}^{V}=\frac{\operatorname{Tr}_{\mathcal{F}_{ \pm}^{V}}\left(A e^{-\beta H_{V}+\beta \mu N}\right)}{\Xi_{ \pm}^{V}(\mu)}
$$

for every bounded operator $A$ on $\mathcal{F}_{ \pm}^{V} . \quad N=N_{V}$ is the operator for the number of particles in the box $V,\left.N\right|_{L_{ \pm}^{2}\left(V^{n}\right)}=n 1_{L_{ \pm}^{2}\left(V^{n}\right)}$, and $\Xi_{ \pm}^{V}(\mu)=$ 
$\operatorname{Tr}_{\mathcal{F}_{ \pm}}\left(e^{-\beta H_{V}+\beta \mu N}\right)$ denotes the partition function. As is well known (see, for example, [B]) we have

$$
\begin{aligned}
& \Xi_{+}^{V}(\mu)=\prod_{k}\left(1-e^{-\beta \epsilon(k)+\beta \mu}\right)^{-1}, \\
& \Xi_{-}^{V}(\mu)=\prod_{k}\left(1+e^{-\beta \epsilon(k)+\beta \mu}\right) .
\end{aligned}
$$

The infinite-volume thermal state is defined through the limit

$$
\langle\cdot\rangle=\lim _{V \nearrow \mathbb{R}^{d}}\langle\cdot\rangle^{V}
$$

when taking averages of local observables [BR, Sec. 2.6].

Taking the infinite volume limit of (2.6) and (2.7) one obtains the grand canonical pressure

$$
p_{\varepsilon}(\mu)=\lim _{V \nearrow \mathbb{R}^{d}} \frac{\log \Xi(\mu)}{\beta|V|}=-\frac{\varepsilon}{\beta(2 \pi)^{d}} \int d^{d} k \log \left(1-\varepsilon e^{-\beta \epsilon(k)+\beta \mu}\right)
$$

and the average density

$$
\rho_{\varepsilon}(\mu)=\frac{d p_{\varepsilon}}{d \mu}(\mu)=\frac{1}{(2 \pi)^{d}} \int d^{d} k \frac{1}{e^{\beta \epsilon(k)-\beta \mu}-\varepsilon} .
$$

$p_{-}$is real analytic on the whole axis, whereas $p_{+}$is real analytic only for $\mu<0$ and has a finite limit as $\mu \rightarrow 0_{-}$. For convenience, we define $p_{+}(\mu)=\infty$ for $\mu>0$. The slope of $p_{+}$at $0_{-}$is related to the Bose-Einstein condensation. We set

$$
\rho_{c}=\rho\left(0_{-}\right)=\frac{1}{(2 \pi)^{d}} \int d^{d} k \frac{1}{e^{\beta \epsilon(k)}-1} .
$$

By the properties of $\epsilon(k), \rho_{c}=\infty$ for $d \leq \gamma$, and is finite otherwise. $\rho_{c}$ is the maximal density of the normal fluid and any surplus density is condensed into the $k=0$ ground state. To simplify the notation we use $\rho_{c}$ also in the case of an ideal Fermi fluid, setting it equal to $\infty$.

The infinite system is assumed to be in a pure thermal state, obtained through the limit (2.8) at the reference chemical potential $\mu$. In this state the average density is $\bar{\rho}=\rho(\mu)<\rho_{c}$. We define the translated pressure by

$$
g_{\varepsilon, \mu}(\lambda)=g_{\varepsilon}(\lambda)=p_{\varepsilon}(\mu+\lambda)-p_{\varepsilon}(\mu) .
$$


$g_{\varepsilon}$ is convex up, increasing, $g(0)=0$, and $g_{\varepsilon}^{\prime}(0)=\bar{\rho}$. For large negative values we have

$$
\lim _{\lambda \rightarrow-\infty} g_{\varepsilon}(\lambda)=-p_{\varepsilon}(\mu), \quad \lim _{\lambda \rightarrow-\infty} g_{\varepsilon}^{\prime}(\lambda)=0,
$$

whereas for positive values

$$
\lim _{\lambda \rightarrow \infty} g_{-}(\lambda)=\infty, \quad \lim _{\lambda \rightarrow \infty} g_{-}^{\prime}(\lambda)=\infty
$$

in the case of fermions and

$$
\lim _{\lambda \rightarrow-\mu} g_{+}(\lambda)=p_{+}(0)-p_{+}(\mu), \quad \lim _{\lambda \rightarrow-\mu} g_{+}^{\prime}(\lambda)=\rho_{c}
$$

for bosons, with $g_{+}(\lambda)=\infty$ for $\lambda>-\mu$.

We define the rate function $f_{\varepsilon}$ as the Legendre transform of $g_{\varepsilon}$, i.e.,

$$
f_{\varepsilon, \mu}(x)=f_{\varepsilon}(x)=\inf _{\lambda \in \mathbb{R}}\left(g_{\varepsilon}(\lambda)-\lambda x\right)=g_{\varepsilon}\left(\lambda_{o}\right)-\lambda_{o} x .
$$

Here $\lambda_{o}=\lambda_{o}(x)$ is the minimizer of $g(\lambda)-\lambda x$, which is unique by convexity. For $x \leq 0$ we have $\lambda_{o}=-\infty$. For $0<x<\rho_{c}$, it is determined by $g^{\prime}\left(\lambda_{o}\right)=x$, while for $x \geq \rho_{c}$ we have $\lambda_{o}=-\mu$. This shows that $f(x)=-\infty$ on the half-line $\{x<0\}$ and $f(x)$ finite elsewhere. In particular, $f$ is convex down, strictly convex for $0<x<\rho_{c}$, and $f_{+}(x)=p(0)-p(\mu)+\mu x$, for $x \geq \rho_{c}$, as a trace of the Bose-Einstein condensation.

Let us now consider a small subvolume $\Lambda$ of our (already infinite) container $V$. The precise shape of $\Lambda$ plays no role, only the "surface area" should be small compared to its volume $|\Lambda|$. Thus, by $\Lambda \nearrow \mathbb{R}^{d}$ we mean a sequence of subdomains such that for each $\Lambda$ there exists a subset $\Lambda^{\prime}$ of $\Lambda$ with $\left|\Lambda^{\prime}\right| /|\Lambda| \rightarrow 1$ and $\operatorname{dist}\left(\Lambda^{\prime}, \mathbb{R}^{d} \backslash \Lambda\right) \rightarrow \infty$.

Let $N_{\Lambda}$ be the number operator for the particles in $\Lambda$. With respect to $\langle\cdot\rangle, N_{\Lambda}$ has some probability distribution. We follow the usual practice and use the same symbol $N_{\Lambda}$ to denote also the corresponding random variable. Its distribution is indicated by $\mathbb{P}$, averages again by $\langle\cdot\rangle$.

We are now in a position to state the main result.

Theorem 2.1 Let $\beta>0$ and $\mu<0$ for BE, resp. $\mu \in \mathbb{R}$ for FD. Consider an interval $I=[a, b]$. Then we have, for $a<\rho_{c}$,

$$
\lim _{\Lambda \nearrow \mathbb{R}^{d}} \frac{1}{\beta|\Lambda|} \log \mathbb{P}\left(\left\{N_{\Lambda} \in|\Lambda| I\right\}\right)=\sup _{x \in I} f_{\varepsilon, \mu}(x)
$$


and, for $a \geq \rho_{c}$,

$$
\limsup _{\Lambda \nearrow \mathbb{R}^{d}} \frac{1}{\beta|\Lambda|} \log \mathbb{P}\left(\left\{N_{\Lambda} \in|\Lambda| I\right\}\right) \leq \sup _{x \in I} f_{\varepsilon, \mu}(x) .
$$

\section{Large deviations in the density}

In this section we explain how Theorem 2.1 follows from the asymptotic behavior of the generating function $\left\langle e^{\beta \lambda N_{\Lambda}}\right\rangle_{\varepsilon}$.

Lemma 3.1 There exists a $\lambda_{\max }(\Lambda)$ such that $\left\langle e^{\beta \lambda N_{\Lambda}}\right\rangle_{\varepsilon}<\infty$ for all $\lambda<$ $\lambda_{\max }(\Lambda)$ and $\left\langle e^{\beta \lambda N_{\Lambda}}\right\rangle_{\varepsilon}=\infty$ for all $\lambda \geq \lambda_{\max }(\Lambda)$. For $F D$ we have $\lambda_{\max }(\Lambda)=$ $\infty$, whereas for $B E \lambda_{\text {max }}(\Lambda)<\infty$ with $\lambda_{\max }(\Lambda) \searrow-\mu$, as $\Lambda \nearrow \mathbb{R}^{d}$.

Theorem 3.2 The limit

$$
\lim _{\Lambda \nearrow \mathbb{R}^{d}} \frac{\log \left\langle e^{\beta \lambda N_{\Lambda}}\right\rangle_{\varepsilon}}{\beta|\Lambda|}=g_{\mu}(\lambda)
$$

including any finite number of derivatives, exists uniformly on compacts of $\mathbb{R}$ for $F D$, resp. of $(-\infty,-\mu)$ for $B E$.

These results are proved in Sections 4 and 5 .

Inferring Theorem 2.1 from our information on the generating function is a standard argument from the theory of large deviations $[\mathrm{E}, \mathrm{O}]$.

The probability of the event in question can be rewritten as

$$
Q_{\Lambda}=\left\langle\chi_{|\Lambda| I}\left(N_{\Lambda}\right)\right\rangle
$$

where $\chi_{A}$ is the indicator function of the set $A \subseteq \mathbb{R}$. To make this event typical we introduce the modified average

$$
\langle\cdot\rangle_{\lambda}=\frac{1}{Z_{\lambda}}\left\langle\cdot e^{\beta \lambda N_{\Lambda}}\right\rangle
$$

where $\lambda<\lambda_{\max }$ and the partition function $Z_{\lambda}=\left\langle e^{\beta \lambda N_{\Lambda}}\right\rangle$. With respect to this new state, (3.18) can be expressed as

$$
Q_{\Lambda}=Z_{\lambda}\left\langle e^{-\beta \lambda N_{\Lambda}} \chi_{|\Lambda| I}\left(N_{\Lambda}\right)\right\rangle_{\lambda}
$$


An upper bound for $Q_{\Lambda}$ comes from the exponential Chebychev inequality,

$$
Q_{\Lambda} \leq\left\langle e^{\beta \lambda\left(N_{\Lambda}-a|\Lambda|\right)}\right\rangle=Z_{\lambda} e^{-\beta \lambda a|\Lambda|}
$$

for any $\lambda<\lambda_{\max }$.

The lower bound requires more effort and can be carried out provided $a<\rho_{c}$. One uses (2.13)-(2.15) to show that there exists a $\lambda_{o}<\lambda_{\max }$ such that $g^{\prime}\left(\lambda_{o}\right)=a$. Differentiating (3.17) twice w.r.t. $\lambda$ we obtain

$$
\begin{gathered}
\lim _{\Lambda \nearrow \mathbb{R}^{d}} \frac{\left\langle N_{\Lambda}\right\rangle_{\lambda_{o}}}{|\Lambda|}=g^{\prime}\left(\lambda_{o}\right)=\rho\left(\mu+\lambda_{o}\right)=a, \\
\lim _{\Lambda \nearrow \mathbb{R}^{d}} \frac{\beta}{|\Lambda|}\left[\left\langle N_{\Lambda}^{2}\right\rangle_{\lambda_{o}}-\left(\left\langle N_{\Lambda}\right\rangle_{\lambda_{o}}\right)^{2}\right]=\frac{d \rho}{d \mu}\left(\mu+\lambda_{o}\right),
\end{gathered}
$$

which is finite. This means that the event $\left\{N_{\Lambda} \approx|\Lambda| a\right\}$ is typical for the new state and a law of large numbers holds. Notice that $\lambda_{o}>0$, since $\rho$ is strictly increasing in $\mu$. From (3.20), $\forall c \in(a, b)$,

$$
\begin{aligned}
Q_{\Lambda} & =Z_{\lambda_{o}}\left\langle e^{-\beta \lambda_{o} N_{\Lambda}} \chi_{|\Lambda|[a, c]}\left(N_{\Lambda}\right)\right\rangle_{\lambda_{o}} \\
& \geq Z_{\lambda_{o}} e^{-\beta \lambda_{o} c|\Lambda|}\left\langle\chi_{|\Lambda|[a, c]}\left(N_{\Lambda}\right)\right\rangle_{\lambda_{o}} \\
& \geq \alpha Z_{\lambda_{o}} e^{-\beta \lambda_{o} c|\Lambda|}
\end{aligned}
$$

for some $\alpha \in(0,1)$ and $|\Lambda|$ large. In fact, $\left\langle\chi_{|\Lambda|[a, c]}\left(N_{\Lambda}\right)\right\rangle_{\lambda_{0}} \rightarrow 1 / 2$ as $\Lambda \nearrow \mathbb{R}^{d}$.

Therefore, when $a<\rho_{c}$, we obtain from (3.21), (3.24) and Theorem 3.2:

$$
g\left(\lambda_{o}\right)-\lambda_{o} c+o(1) \leq \frac{\log Q_{\Lambda}}{\beta|\Lambda|} \leq g\left(\lambda_{o}\right)-\lambda_{o} a+o(1)
$$

Since $c \in(a, b)$ is arbitrary, we conclude that

$$
\lim _{\Lambda \nearrow \mathbb{R}^{d}} \frac{\log Q_{\Lambda}}{\beta|\Lambda|}=g\left(\lambda_{o}\right)-\lambda_{o} a=f(a)=\sup _{x \in[a, b]} f(x),
$$

where $f$ is the rate function defined in (2.16). $\lambda_{o}$ is the same as in the definition of the Legendre transform (2.16), because of (3.22). The last equality comes from the convexity of $f$.

The second assertion of the theorem is now easy, since the right inequality in (3.25) holds without any restriction in $a$.

Q.E.D.

Theorem 2.1 implies the central limit theorem for the density in $\Lambda$. 
Corollary 3.3 Under the assumptions of Theorem 2.1, the moments of the variable $\xi_{\Lambda}=\left(N_{\Lambda}-\left\langle N_{\Lambda}\right\rangle\right) /|\Lambda|^{1 / 2}$ converge, as $\Lambda \nearrow \mathbb{R}^{d}$, to those of a Gaussian with variance $\beta^{-1}(d \rho / d \mu)(\mu)$.

Proof. The $k$-th cumulant of $\xi_{\Lambda}$ is given by

$$
C_{\Lambda}(k)=\frac{1}{\beta^{k}|\Lambda|^{k / 2}}\left[\frac{d^{k}}{d \lambda^{k}} \log \left\langle e^{\beta \lambda N_{\Lambda}}\right\rangle\right]_{\lambda=0},
$$

$k \geq 2$. From Theorem 3.2, $C_{\Lambda}(2) \rightarrow \beta^{-1} g^{\prime \prime}(0)=\beta^{-1}(d \rho / d \mu)(\mu)$, whereas, for $k>2, C_{\Lambda}(k) \rightarrow 0$. Also $C_{\Lambda}(1)=0$. These limits are the cumulants of a centered Gaussian variable with the specified variance.

Q.E.D.

\section{Generating function}

We derive a determinant formula for the generating function $\left\langle e^{\beta \lambda N_{\Lambda}}\right\rangle_{\varepsilon}$. With its help we prove the claims of Lemma 3.1. We will see in the next section that is convenient to introduce the variables $\zeta=e^{\beta \lambda}$ and $\widetilde{\zeta}=\zeta-1$.

By (2.4), we have

$$
\begin{aligned}
-\beta H_{V}+\beta \mu N_{V}=\left\langle\mathbf{a}\left|\left(-\beta h_{V}+\beta \mu 1_{V}\right)\right| \mathbf{a}\right\rangle & =\left\langle\mathbf{a}\left|A_{V}\right| \mathbf{a}\right\rangle, \\
\beta \lambda N_{\Lambda}=\left\langle\mathbf{a}\left|\beta \lambda \chi_{\Lambda}\right| \mathbf{a}\right\rangle & =\left\langle\mathbf{a}\left|B_{\Lambda}\right| \mathbf{a}\right\rangle,
\end{aligned}
$$

which implicitly define $A_{V}$ and $B_{\Lambda}$ as linear operators on $L^{2}(V)$. We will use the following identity.

Lemma 4.1 Let $A, B$ be self-adjoint and bounded from above. Then there exists a self-adjoint operator $C$ such that $e^{A} e^{B} e^{A}=e^{C}$ and

$$
e^{\langle\mathbf{a}|A| \mathbf{a}\rangle} e^{\langle\mathbf{a}|B| \mathbf{a}\rangle} e^{\langle\mathbf{a}|A| \mathbf{a}\rangle}=e^{\langle\mathbf{a}|C| \mathbf{a}\rangle}
$$

for both BE and FD.

Proof. See Appendix.

We apply Lemma 4.1 with $A=A_{V} / 2$ and $B=B_{\Lambda}$, after a symmetrization of the density matrix in (2.5). Then, using also definition (2.8),

$$
\left\langle e^{\beta \lambda N_{\Lambda}}\right\rangle=\lim _{V \nearrow \mathbb{R}^{d}} \frac{\operatorname{Tr}_{\mathcal{F} V}\left(e^{\langle\mathbf{a}|C| \mathbf{a}\rangle}\right)}{\operatorname{Tr}_{\mathcal{F}^{V}}\left(e^{\left\langle\mathbf{a}\left|A_{V}\right| \mathbf{a}\right\rangle}\right)} .
$$


Evaluating the trace of a quadratic form in $a_{i}^{*}, a_{j}$ is a standard calculation for both BE and FD. Let us consider first the case of fermions. For a self-adjoint operator $A$ on $L^{2}(V)$ such that $e^{A}$ is trace-class, we have

$$
\operatorname{Tr}_{\mathcal{F}_{-}}\left(e^{\langle\mathbf{a}|A| \mathbf{a}\rangle}\right)=\operatorname{det}_{V}\left(1_{V}+e^{A}\right)=\operatorname{det}\left(1+\chi_{V} e^{A} \chi_{V}\right),
$$

where $\operatorname{det}_{V}$ is the determinant on $L^{2}(V)$ and det the determinant on $L^{2}\left(\mathbb{R}^{d}\right)$. Here and in the sequel we refer to the theory of infinite determinants, as found, e.g., in [RS, Sec. XIII.17]. $e^{A_{V}}$ is obviously trace-class, and so is $e^{C}$, since $e^{B_{\Lambda}}$ is bounded. Using the definition of $C$, we obtain

$$
\begin{aligned}
\frac{\operatorname{det}_{V}\left(1_{V}+e^{C}\right)}{\operatorname{det}_{V}\left(1_{V}+e^{A_{V}}\right)} & =\operatorname{det}_{V}\left[\left(1_{V}+e^{A_{V}}\right)^{-1}\left(1_{V}+e^{A_{V} / 2} e^{B_{\Lambda}} e^{A_{V} / 2}\right)\right] \\
& =\operatorname{det}_{V}\left[1_{V}+\left(1_{V}+e^{A_{V}}\right)^{-1} e^{A_{V} / 2}\left(e^{B_{\Lambda}}-1_{V}\right) e^{A_{V} / 2}\right] \\
& =\operatorname{det}\left[1+\widetilde{\zeta} \chi_{\Lambda} D_{V,-} \chi_{\Lambda}\right],
\end{aligned}
$$

where $D_{V,-}=\left(1+e^{A_{V}}\right)^{-1} e^{A_{V}}$. We used the fact that $e^{B_{\Lambda}}=\left(e^{\beta \lambda}-1\right) \chi_{\Lambda}+1=$ $\widetilde{\zeta} \chi_{\Lambda}+1$ and the cyclicity of the trace in the definition of the determinant. Finally, from (4.3) and (4.5),

$$
\left\langle e^{\beta \lambda N_{\Lambda}}\right\rangle_{-}=\lim _{V \nearrow \mathbb{R}^{d}} \operatorname{det}\left[1+\widetilde{\zeta} \chi_{\Lambda} D_{V,-} \chi_{\Lambda}\right]
$$

One would like to take the limit on $V$ inside the determinant by replacing $D_{V,-}$ with the corresponding operator on $L^{2}\left(\mathbb{R}^{d}\right)$ defined as

$$
\widehat{\left(D_{-} \psi\right)}(k)=\widehat{d_{-}}(k) \widehat{\psi}(k), \quad\left(D_{-} \psi\right)(x)=\int d y d_{-}(y-x) \psi(y),
$$

where ${ }^{\wedge}$ denotes the Fourier transform and

$$
\widehat{d_{-}}(k)=\frac{1}{1+e^{\beta(\epsilon(k)-\mu)}} .
$$

Notice that $\widehat{d_{-}} \in L^{1}\left(\mathbb{R}^{d}\right)$ by our assumptions on $\epsilon(k)$ and so $d_{-} \in L^{\infty}\left(\mathbb{R}^{d}\right)$. Moreover, (2.3) ensures that $d_{-} \in L^{1}\left(\mathbb{R}^{d}\right)$.

By [RS, Sec. XIII.17, Lemma 4(d)] one has to establish that $\chi_{\Lambda} D_{V,-} \chi_{\Lambda}$ tends to $\chi_{\Lambda} D_{-} \chi_{\Lambda}$ in the trace norm. 
Lemma 4.2 Let $\widehat{d}$ be a continuous integrable function on $\mathbb{R}^{d}$. We define $D$ through (4.7) as a linear operator acting on $L^{2}\left(\mathbb{R}^{d}\right)$. Furthermore we define $D_{V}$ by $D_{V}|k\rangle=\widehat{d}(k)|k\rangle$ on $L^{2}(V)$ and by $D_{V}=0$ on the orthogonal complement $L^{2}\left(\mathbb{R}^{d} \backslash V\right)$. Then, for $\Lambda \subset V, \chi_{\Lambda} D_{V} \chi_{\Lambda}$ and $\chi_{\Lambda} D \chi_{\Lambda}$ are traceclass, and

$$
\lim _{V \nearrow \mathbb{R}^{d}} \operatorname{Tr}\left|\chi_{\Lambda}\left(D_{V}-D\right) \chi_{\Lambda}\right|=0
$$

Proof. See Appendix.

We conclude that

$$
\left\langle\zeta^{N_{\Lambda}}\right\rangle_{-}=\operatorname{det}\left(1+\widetilde{\zeta} \chi_{\Lambda} D_{-} \chi_{\Lambda}\right)
$$

with $\widetilde{\zeta}=\zeta-1$.

For bosons we proceed in the same way, except that (4.4) is replaced by

$$
\operatorname{Tr}_{\mathcal{F}_{+}^{V}}\left(e^{\langle\mathbf{a}|A| \mathbf{a}\rangle}\right)=\operatorname{det}_{V}\left(1_{V}-e^{A}\right)^{-1},
$$

requiring in addition $\left\|e^{A}\right\|<1$. In fact, for $\left\|e^{A}\right\| \geq 1$, the l.h.s. of (4.10) is $\infty$, whereas the r.h.s. might be finite if 1 is not an eigenvalue of the trace-class operator $e^{A}$. In our case, by assumption $\left\|e^{A_{V}}\right\|<1$. As for $e^{C}$, the function

$$
\lambda \mapsto\left\|e^{C}\right\|=\left\|\left(e^{\beta \lambda}-1\right) e^{A_{V} / 2} \chi_{\Lambda} e^{A_{V} / 2}+e^{A_{V}}\right\|
$$

is increasing and $\lambda_{\max }(\Lambda)$ is defined to be that $\lambda$ which makes it equal to 1 . Since the r.h.s. of (4.11) is increasing in $\Lambda$ and its sup is $e^{\beta \lambda}\left\|e^{A_{V}}\right\|=e^{\beta(\lambda+\mu)}$, then one checks that $\lambda_{\max }(\Lambda) \searrow-\mu$, as $\Lambda \nearrow \mathbb{R}^{d}$. Therefore, following the computation for FD, we have

$$
\left\langle\zeta^{N_{\Lambda}}\right\rangle_{+}=\lim _{V \nearrow \mathbb{R}^{d}} \frac{\operatorname{det}\left(1-e^{C}\right)^{-1}}{\operatorname{det}\left(1-e^{A_{V}}\right)^{-1}}=\operatorname{det}\left(1+\widetilde{\zeta} \chi_{\Lambda} D_{+} \chi_{\Lambda}\right)^{-1}
$$

for $\lambda<\lambda_{\max }$ and $\infty$ otherwise. Here $D_{+}$, the limit of $D_{V,+}=\left(e^{A_{V}}-1\right)^{-1} e^{A_{V}}$, is defined as in (4.7) with

$$
\widehat{d_{+}}(k)=\frac{1}{1-e^{\beta(\epsilon(k)-\mu)}} .
$$

Equation (4.12) is the analogue of (4.9) and proves Lemma 3.1 . 


\section{$5 \quad$ Infinite volume limit}

Instead of the chemical potential, in this section we use the fugacity $z=e^{\beta \mu}$, regarding it as a complex variable. This will come out handy for the proof of Theorem 3.2. The variables $\zeta$ and $\widetilde{\zeta}$, defined at the beginning of the previous section, will also be extended to the complex plane. In this setup the translated pressure (2.12) becomes

$$
g_{z}(\zeta)=p(z \zeta)-p(z)
$$

where, with a slight abuse of notation, we keep the same name for the pressure as a function of the fugacity.

Expressions (2.9)-(2.10) for the pressure and the average density define two analytic functions of $\mu$ in

$$
\begin{aligned}
& E_{+}=\{\operatorname{Re} \mu<0\} \cup\{\operatorname{Re} \mu \geq 0, \operatorname{Im} \mu \neq 2 \pi j / \beta, \forall j \in \mathbb{Z}\}, \\
& E_{-}=\{\operatorname{Re} \mu<0\} \cup\{\operatorname{Re} \mu \geq 0, \operatorname{Im} \mu \neq(2 j+1) \pi / \beta, \forall j \in \mathbb{Z}\} .
\end{aligned}
$$

Hence $g_{\varepsilon}(\zeta)$ is analytic in

$$
G_{+}=\mathbb{C} \backslash\left[z^{-1},+\infty\right) ; \quad G_{-}=\mathbb{C} \backslash\left(-\infty,-z^{-1}\right]
$$

We proceed to give the proof of Theorem 3.2. Let $K \subset G_{\varepsilon}$ be a compact set in the complex plane. We choose $K$ such that $L=K \cap \mathbb{R}^{+}$is also compact, since its image through the function $\zeta \mapsto \lambda$ verifies the hypotheses of the theorem. Our argument, however, is valid for any $K$. Without loss of generality, we can assume that $1 \in K$.

For $\widetilde{\zeta}$ restricted to $G_{\varepsilon} \cap \mathbb{R}^{+}$, let us define

$$
\phi_{\varepsilon, z}^{\Lambda}(\zeta)=\frac{1}{|\Lambda|} \log \left\langle\zeta^{N_{\Lambda}}\right\rangle_{\varepsilon, z}=-\frac{\varepsilon}{|\Lambda|} \operatorname{Tr} \log \left(1+\widetilde{\zeta} \chi_{\Lambda} D_{\varepsilon} \chi_{\Lambda}\right)
$$

according to (4.9) and (4.12). The proof of Theorem 3.2 will be subdivided into three steps.

1. $\phi^{\Lambda}$ can be analytically continued to $G_{\varepsilon}$.

2. There is a positive $r$ such that $\phi^{\Lambda}(\zeta)$ converges uniformly to $\beta g_{z}(\zeta)$ for $|\zeta-1| \leq r$. 
3. $\left|\phi^{\Lambda}\right|$ is uniformly bounded on $K$. Therefore by Vitali's lemma [Ti, Sec. 5.21] $\left|\phi^{\Lambda}\right|$ and any finite number of its derivatives converge uniformly on $K$.

SteP 1. We leave the proof of the following lemma for the Appendix.

Lemma 5.1 The function $\phi_{\varepsilon}^{\Lambda}(\zeta)$, as defined by the trace in (5.5), is analytic in $G_{\varepsilon}$.

STEP 2. Expanding the log in (5.5) one has, for $|\widetilde{\zeta}|<\left\|D_{\varepsilon}\right\|^{-1}$,

$$
\phi_{\varepsilon}^{\Lambda}(\widetilde{\zeta}+1)=-\frac{\varepsilon}{|\Lambda|} \operatorname{Tr} \sum_{m=1}^{\infty} \frac{(-1)^{m-1}}{m}\left(\widetilde{\zeta} \chi_{\Lambda} D_{\varepsilon} \chi_{\Lambda}\right)^{m} .
$$

We would like to interchange the summation with the trace. To do so, we need dominated convergence for the series:

$$
\left|\widetilde{\zeta} \chi_{\Lambda} D_{\varepsilon} \chi_{\Lambda}\right|^{m} \leq|\widetilde{\zeta}|^{m}\left\|D_{\varepsilon}\right\|^{m-1}\left(\chi_{\Lambda} D_{\varepsilon} \chi_{\Lambda}\right) .
$$

Since $|\Lambda|^{-1} \operatorname{Tr}\left(\chi_{\Lambda} D_{\varepsilon} \chi_{\Lambda}\right)=d_{\varepsilon}(0)$ (see proof of Lemma 1.2 in the Appendix), each term of (5.6) is bounded by a term of an integrable series independent of $\Lambda$. Therefore, for the same $\widetilde{\zeta}$ 's as above,

$$
\phi_{\varepsilon}^{\Lambda}(\widetilde{\zeta}+1)=-\varepsilon \sum_{m=1}^{\infty} \frac{(-1)^{m-1} \widetilde{\zeta}^{m}}{m} \frac{1}{|\Lambda|} \operatorname{Tr}\left(\chi_{\Lambda} D_{\varepsilon} \chi_{\Lambda}\right)^{m} .
$$

Suppose that we are able to prove that

$$
\lim _{\Lambda \nearrow \mathbb{R}^{d}} \frac{1}{|\Lambda|} \operatorname{Tr}\left(\chi_{\Lambda} D_{\varepsilon} \chi_{\Lambda}\right)^{m}=\int d k\left[\widehat{d}_{\varepsilon}(k)\right]^{m},
$$

with a rest bounded above by $m R^{m}$ for some positive constant $R$. Then, using (4.8) and (4.13), we would have that, for any $r<\min \left\{\left\|D_{\varepsilon}\right\|^{-1}, R^{-1}\right\}$, uniformly for $|\widetilde{\zeta}| \leq r$,

$$
\begin{aligned}
\lim _{\Lambda \nearrow \mathbb{R}^{d}} \phi_{\varepsilon}^{\Lambda}(\widetilde{\zeta}+1) & =-\varepsilon \sum_{m=1}^{\infty} \frac{(-1)^{m-1} \widetilde{\zeta}^{m}}{m} \int d k\left(\frac{1}{1-\varepsilon z^{-1} e^{\beta \epsilon(k)}}\right)^{m} \\
& =-\varepsilon \int d k \log \left(1+\frac{\zeta-1}{1-\varepsilon z^{-1} e^{\beta \epsilon(k)}}\right) \\
& =-\varepsilon \int d k \log \left(\frac{1-\varepsilon z \zeta e^{-\beta \epsilon(k)}}{1-\varepsilon z e^{-\beta \epsilon(k)}}\right)=\beta g_{z}(\widetilde{\zeta}+1),
\end{aligned}
$$


the last equality coming from (2.9). This would complete Step 2.

Let us pursue this project. One sees that

$$
\begin{gathered}
\int d k\left[\widehat{d}_{\varepsilon}(k)\right]^{m}=\underbrace{\left(d_{\varepsilon} * d_{\varepsilon} * \cdots * d_{\varepsilon}\right)}_{m \text { times }}(0) \\
=\frac{1}{|\Lambda|} \int_{\Lambda} d x_{1} \int_{\mathbb{R}^{d}} d x_{2} d_{\varepsilon}\left(x_{1}-x_{2}\right) \cdots \int_{\mathbb{R}^{d}} d x_{m} d_{\varepsilon}\left(x_{m-1}-x_{m}\right) d_{\varepsilon}\left(x_{m}-x_{1}\right) .
\end{gathered}
$$

The normalized integration over $x_{1}$ is harmless since, by translation invariance, the integrand does not depend on that variable. On the other hand, it is not hard to verify that

$$
\begin{gathered}
\frac{1}{|\Lambda|} \operatorname{Tr}\left(\chi_{\Lambda} D_{\varepsilon} \chi_{\Lambda}\right)^{m}=\frac{1}{|\Lambda|} \int_{\Lambda} d x_{1}\left\langle x_{1}\left|\left(\chi_{\Lambda} D_{\varepsilon} \chi_{\Lambda}\right)^{m}\right| x_{1}\right\rangle \\
=\frac{1}{|\Lambda|} \int_{\Lambda} d x_{1} \int_{\Lambda} d x_{2} d_{\varepsilon}\left(x_{1}-x_{2}\right) \cdots \int_{\Lambda} d x_{m} d_{\varepsilon}\left(x_{m-1}-x_{m}\right) d_{\varepsilon}\left(x_{m}-x_{1}\right) .
\end{gathered}
$$

In view of (5.9), we want to compare (5.11) with (5.12). We observe that

$$
\underbrace{\int_{\mathbb{R}^{d}} \int_{\mathbb{R}^{d}} \cdots \int_{\mathbb{R}^{d}}}_{m-1 \text { times }}-\underbrace{\int_{\Lambda} \int_{\Lambda} \cdots \int_{\Lambda}}_{m-1 \text { times }}=\sum_{i=1}^{m-1} \underbrace{\int_{\Lambda} \cdots \int_{\Lambda}}_{i-1 \text { times }} \int_{\Lambda^{c}} \underbrace{\int_{\mathbb{R}^{d}} \cdots \int_{\mathbb{R}^{d}}}_{m-1-i \text { times }} .
$$

Subtracting (5.11) from (5.12) leads then to $m-1$ terms of the form

$$
\frac{1}{|\Lambda|} \int_{\Lambda} d x_{1} \int_{\Lambda^{c}} d x_{2} d_{\varepsilon}\left(x_{1}-x_{2}\right) \cdots \int_{A_{m}} d x_{m} d_{\varepsilon}\left(x_{m-1}-x_{m}\right) d_{\varepsilon}\left(x_{m}-x_{1}\right),
$$

where the sets $A_{3}, \ldots, A_{m}$ can be either $\mathbb{R}^{d}$ or $\Lambda$. (5.14) holds because, due to the cyclicity of the integration variables, one can cyclically permute the order of integration without touching the integrand. We overestimate by switching to absolute values and integrating $x_{3}, \ldots, x_{m}$ over $\mathbb{R}^{d}$,

$$
\left.\frac{1}{|\Lambda|} \int_{\Lambda} d x_{1}\left(\chi_{\Lambda^{c}-x_{1}}\left|d_{\varepsilon}\right|\right) *\left|d_{\varepsilon}\right| * \cdots *\left|d_{\varepsilon}\right|\right)\left(x_{1}\right)=\frac{1}{|\Lambda|} \int_{\Lambda} d x_{1} u_{\Lambda}\left(x_{1}\right),
$$

which defines $u_{\Lambda}\left(x_{1}\right)$. To estimate this function, we use recursively the relation $\|f * g\|_{\infty} \leq\|f\|_{\infty}\|g\|_{1}$ and obtain

$$
u_{\Lambda}\left(x_{1}\right) \leq\left\|d_{\varepsilon}\right\|_{1}^{m-1} \sup _{\Lambda^{c}-x_{1}}\left|d_{\varepsilon}\right| .
$$


Recalling now the definition of $\Lambda^{\prime}$ given before the statement of Theorem 2.1, one sees that, if $x_{1} \in \Lambda^{\prime}$ and $y \in \Lambda^{c}-x_{1}$, then $|y| \rightarrow \infty$ as $\Lambda \nearrow \mathbb{R}^{d}$. Hence, from (5.16),

$$
\sup _{x_{1} \in \Lambda^{\prime} m \geq 1} \sup _{m \geq 1}\left\|d_{\varepsilon}\right\|_{1}^{-m+1} u_{\Lambda}\left(x_{1}\right) \rightarrow 0 .
$$

Also from (5.16), pointwise in $x_{1}$,

$$
\sup _{m \geq 1}\left\|d_{\varepsilon}\right\|_{1}^{-m+1} u_{\Lambda}\left(x_{1}\right) \leq\left\|d_{\varepsilon}\right\|_{\infty} .
$$

When we average over $x_{1} \in \Lambda$, the last two relations and the properties of $\Lambda^{\prime}$ prove that

$$
\lim _{\Lambda \nearrow \mathbb{R}^{d}} \sup _{m \geq 1}\left\|d_{\varepsilon}\right\|_{1}^{-m+1} \frac{1}{|\Lambda|} \int_{\Lambda} d x_{1} u_{\Lambda}\left(x_{1}\right)=0 .
$$

This takes care of each term as in (5.14), and we have $m-1$ of these terms. Hence (5.9) holds with $R=\left\|d_{\varepsilon}\right\|_{1}$. This ends Step 2.

STEP 3. Again we expand (5.5) in powers of $\widetilde{\zeta}$, but this time about a generic $\widetilde{\zeta}_{0} \notin G_{\varepsilon}-1$ (see (5.4)). We obtain

$$
\begin{aligned}
& \frac{1}{|\Lambda|} \operatorname{Tr} \log \left(1+\widetilde{\zeta} \chi_{\Lambda} D_{\varepsilon} \chi_{\Lambda}\right)=\frac{1}{|\Lambda|} \operatorname{Tr} \log \left(1+\widetilde{\zeta}_{0} \chi_{\Lambda} D_{\varepsilon} \chi_{\Lambda}\right) \\
+ & \frac{1}{|\Lambda|} \operatorname{Tr} \sum_{m=1}^{\infty} \frac{(-1)^{m-1}}{m}\left(\left(1+\widetilde{\zeta}_{0} \chi_{\Lambda} D_{\varepsilon} \chi_{\Lambda}\right)^{-1} \chi_{\Lambda} D_{\varepsilon} \chi_{\Lambda}\right)^{m}\left(\widetilde{\zeta}-\widetilde{\zeta}_{0}\right)^{m}
\end{aligned}
$$

Let us estimate this series. First of all, using some spectral theory $\mathbb{W}$, Sec. 7.4],

$$
\begin{aligned}
\left\|\left(1+\widetilde{\zeta}_{0} \chi_{\Lambda} D_{\varepsilon} \chi_{\Lambda}\right)^{-1}\right\| & \leq\left[\operatorname{dist}\left(1, \sigma\left(-\widetilde{\zeta}_{0} \chi_{\Lambda} D_{\varepsilon} \chi_{\Lambda}\right)\right)\right]^{-1} \\
& \leq\left[\operatorname{dist}\left(1, \sigma\left(-\widetilde{\zeta}_{0} D_{\varepsilon}\right)\right)\right]^{-1}
\end{aligned}
$$

since we know from definitions (4.7), (4.8) and (4.13) that

$$
\begin{aligned}
\sigma\left(\chi_{\Lambda} D_{-} \chi_{\Lambda}\right) & \subset\left[0,\left\|\chi_{\Lambda} D_{-} \chi_{\Lambda}\right\|\right] \subset\left[0,\left\|D_{-}\right\|\right] \\
& =\sigma\left(D_{-}\right)=\left[0,1 /\left(1+z^{-1}\right)\right], \\
\sigma\left(\chi_{\Lambda} D_{+} \chi_{\Lambda}\right) & \subset\left[-\left\|\chi_{\Lambda} D_{+} \chi_{\Lambda}\right\|, 0\right] \subset\left[-\left\|D_{+}\right\|, 0\right] \\
& =\sigma\left(D_{+}\right)=\left[1 /\left(1-z^{-1}\right), 0\right] .
\end{aligned}
$$


Repeating the same reasoning as in Step 2, we use the above to exchange the trace with the summation in (5.20) - which is legal for small $\left|\widetilde{\zeta}-\widetilde{\zeta}_{0}\right|$ as to be determined shortly. This yields a new series, whose $m$-th term is bounded above by

$$
\left[\operatorname{dist}\left(1, \sigma\left(-\widetilde{\zeta}_{0} D_{\varepsilon}\right)\right)\right]^{-m}\left\|D_{\varepsilon}\right\|^{m-1} d_{\varepsilon}(0)\left|\widetilde{\zeta}-\widetilde{\zeta}_{0}\right|^{m}=a\left(b\left(\widetilde{\zeta}_{0}\right)\left|\widetilde{\zeta}-\widetilde{\zeta}_{0}\right|\right)^{m}
$$

where $d_{\varepsilon}(0)=|\Lambda|^{-1} \operatorname{Tr}\left(\chi_{\Lambda} D_{\varepsilon} \chi_{\Lambda}\right)$. Hence, in view of (5.5), (5.20) implies

$$
\left|\phi_{\varepsilon}^{\Lambda}(\widetilde{\zeta}+1)\right| \leq\left|\phi_{\varepsilon}^{\Lambda}\left(\widetilde{\zeta}_{0}+1\right)\right|+a \frac{b\left(\widetilde{\zeta}_{0}\right)\left|\widetilde{\zeta}-\widetilde{\zeta}_{0}\right|}{1-b\left(\widetilde{\zeta}_{0}\right)\left|\widetilde{\zeta}-\widetilde{\zeta}_{0}\right|} \leq\left|\phi_{\varepsilon}^{\Lambda}\left(\widetilde{\zeta}_{0}+1\right)\right|+a,
$$

for $\left|\widetilde{\zeta}-\widetilde{\zeta}_{0}\right| \leq\left(2 b\left(\widetilde{\zeta}_{0}\right)\right)^{-1}$

The crucial fact is that $b(\widetilde{\zeta})^{-1}$ stays away from zero when $\widetilde{\zeta}$ is away from the boundary of $G_{\varepsilon}-1$. This can be seen via the following argument, exploiting (5.24) and (5.22)-(5.23). In the FD case $\sigma\left(-\widetilde{\zeta}_{0} D_{-}\right)$is a segment that has one endpoint at the origin and the phase of $-\widetilde{\zeta}_{0}$ is the angle it forms with the positive semi-axis. This means that, as long as $\widetilde{\zeta}_{0}$ does not go anywhere near the negative semi-axis, we are safe. For $\widetilde{\zeta}_{0} \in\left(-z^{-1}-1,0\right)$ (see $(5.4)), \sigma\left(-\widetilde{\zeta}_{0} D_{-}\right)$is contained in $\mathbb{R}_{o}^{+}$. However, notice from (5.22) that the other endpoint is located at $-\widetilde{\zeta}_{0} /\left(1+z^{-1}\right)<1$. For BE the reasoning is analogous, except that in this case the phase of $\widetilde{\zeta}_{0}$ is the angle between $\sigma\left(-\widetilde{\zeta}_{0} D_{+}\right)$and $\mathbb{R}_{o}^{+}$. Therefore the "safe" span is the complement of the positive semi-axis. Also, if $\widetilde{\zeta}_{0} \in\left(0, z^{-1}-1\right)$ (again see (5.4)), the "floating" endpoint of $\sigma\left(-\widetilde{\zeta}_{0} D_{+}\right)$is found at $\widetilde{\zeta}_{0} /\left(z^{-1}-1\right)<1$.

With the above estimate we can use (5.25) recursively. If $\left|\widetilde{\zeta}_{0}\right| \leq r$, from Step $2,\left|\phi_{\varepsilon}^{\Lambda}\left(\widetilde{\zeta}_{0}+1\right)\right| \leq M$, for some $M$, since $\phi_{\varepsilon}^{\Lambda}$ converges uniformly there. Then, from (5.25), we have that $\left|\phi_{\varepsilon}^{\Lambda}\left(\widetilde{\zeta}_{1}+1\right)\right| \leq M+a$, for any $\widetilde{\zeta}_{1}$ such that $\left|\widetilde{\zeta}_{1}-\widetilde{\zeta}_{0}\right|<\left(2 b\left(\widetilde{\zeta}_{0}\right)\right)^{-1}$. Proceeding, we see that $\left|\phi_{\varepsilon}^{\Lambda}\left(\widetilde{\zeta}_{k}+1\right)\right| \leq M+k a$, whenever $\left|\widetilde{\zeta}_{k}-\widetilde{\zeta}_{k-1}\right|<\left(2 b\left(\widetilde{\zeta}_{k-1}\right)\right)^{-1}$. In this way we will cover $K$ in finitely many steps since it keeps at a certain distance from the boundary of $G_{\varepsilon}$ and the $\left(b\left(\widetilde{\zeta}_{k}\right)\right)^{-1}$ are bounded below. This completes Step 3, i.e., $\phi_{\varepsilon}^{\Lambda}(\zeta)$ is bounded on $K$ and Vitali's lemma can be applied.

Q.E.D.

Acknowledgements. We thank S. Olla and G. Gallavotti for very instructive discussions. Work at Rutgers was supported by NSF Grant DMR9813268. 


\section{A Appendices}

\section{A.1 Relativistic massless particles}

We prove that the energy dispersion $\epsilon(k)=c|k|$ satisfies our assumptions. The only condition to be checked is $(2.3)$, that is, the Fourier transform of $k \mapsto\left(e^{\beta(c|k|-\mu)}-\varepsilon\right)^{-1}$ is in $L^{1}\left(\mathbb{R}^{d}\right)$. This is a consequence of the following

Lemma A.1 Let $f:[0,+\infty) \longrightarrow \mathbb{C}$ be of Schwartz class. With the common abuse of notation, denote by $\widehat{f}(|\xi|)$ the Fourier transform of $f(|x|)$, for $x, \xi \in$ $\mathbb{R}^{d}$. Then, for some positive $C$,

$$
\widehat{f}(|\xi|) \leq \frac{C}{|\xi|^{d+1}}
$$

Proof. For simplicity let us write $\xi=|\xi|$. The Fourier transform of a radial function is

$$
\widehat{f}(\xi)=\frac{(2 \pi)^{d / 2}}{\xi^{d / 2-1}} \int_{0}^{\infty} d r f(r) r^{d / 2} J_{d / 2-1}(r \xi),
$$

cf. [SW, Chap. IV, Th. 3.3], where $J_{\nu}$ is the standard Bessel function of order $\nu$ Wa. One has

$$
J_{\nu}(x) \approx \frac{x^{\nu}}{2^{\nu} \Gamma(\nu+1)}
$$

for $x \rightarrow 0$, whereas

$$
J_{\nu}(x)=\sqrt{\frac{2}{\pi x}}\left[\cos \left(x-\frac{2 \nu+1}{4} \pi\right)+g(x)\right],
$$

with $g(x) \rightarrow 0$ for $x \rightarrow \infty$. Using the relation

$$
\int_{0}^{x} d t t^{\nu} J_{\nu-1}(t)=x^{\nu} J_{\nu}(x)
$$

we integrate (A.1) by parts repeatedly, taking into account also (A.2) and the hypothesis on $f$. After $n$ integrations we get, up to constants, $n$ terms of the form

$$
\frac{1}{\xi^{d / 2+n-1}} \int_{0}^{\infty} d r f^{(i)}(r) r^{d / 2-n+i} J_{d / 2+n-1}(r \xi)
$$

with $i=1, \ldots, n$. For our purposes it suffices to iterate up to $n \geq d / 2+2$. In fact, if $i$ is such that $d / 2-n+i>-d$, then in (A.5) we can estimate the 
Bessel function by a constant. The integral converges by the rapid decay of $f^{(i)}$ and the whole term is of the order $\xi^{-d-1}$ or better. For smaller values of $i$, the estimate uses (A.2), for $x \in[0, a]$, and (A.3) otherwise. Since $\left|f^{(i)}\right| \leq c$, A.5) is bounded by

$$
\begin{gathered}
\frac{A}{\xi^{d / 2+n-1}} \int_{0}^{a / \xi} d r r^{d / 2-n+i}(r \xi)^{d / 2+n-1}+ \\
+\frac{B}{\xi^{d / 2+n-1}} \int_{a / \xi}^{\infty} d r r^{d / 2-n+i}(r \xi)^{-1 / 2} \approx \frac{1}{\xi^{d+i}}
\end{gathered}
$$

the second integral being convergent because of the choice of $i$.

Q.E.D.

\section{A.2 Proof of Lemma 4.1}

As before, we set $\varepsilon= \pm 1$, according to either bosons or fermions. A general $f \in L^{2}(V)$ can be expanded in the Fourier basis as $f=\sum_{k} f_{k}|k\rangle$. The corresponding creation operator is then defined by

$$
a(f)^{*}=\sum_{k \in V^{\prime}} f_{k} a_{k}^{*}
$$

For the sake of simplicity, we denote $\mathcal{A}=\langle\mathbf{a}|A| \mathbf{a}\rangle=\sum_{i j} A_{i j} a_{i}^{*} a_{j}$ (same for $\mathcal{B})$. Recalling the canonical (anti)commutation relations,

$$
\begin{aligned}
& {\left[a_{i}, a_{j}^{*}\right]_{-\varepsilon}=a_{i} a_{j}^{*}-\varepsilon a_{j}^{*} a_{i}=\delta_{i j}} \\
& {\left[a_{i}, a_{j}\right]_{-\varepsilon}=a_{i} a_{j}-\varepsilon a_{j} a_{i}=0}
\end{aligned}
$$

one calculates that

$$
\left[\mathcal{A}, a(f)^{*}\right]=a(A f)^{*}
$$

and in exponential form

$$
e^{t \mathcal{A}} a(f)^{*} e^{-t \mathcal{A}}=a\left(e^{t A} f\right)^{*} .
$$

Now, let $|0\rangle$ be the ground state of $\mathcal{F}^{V}$. For $n \in \mathbb{N}$, and $f_{1}, f_{2}, \ldots, f_{n} \in$ $L^{2}(V)$, the finite linear combinations of the states

$$
\left|f_{1}, f_{2}, \ldots, f_{n}\right\rangle=a\left(f_{1}\right)^{*} a\left(f_{2}\right)^{*} \cdots a\left(f_{n}\right)^{*}|0\rangle
$$

are dense in $\mathcal{F}$, which is another way of stating that $|0\rangle$ is cyclic w.r.t. the algebra generated by the creation operators. Therefore, we need only test 
our assertion on vectors of the type (A.11). Using (A.10) with $t=1$, and observing that $\mathcal{A}|0\rangle=0$, we obtain

$$
\begin{aligned}
e^{\mathcal{A}}\left|f_{1}, \ldots, f_{n}\right\rangle & =e^{\mathcal{A}} a\left(f_{1}\right)^{*} e^{-\mathcal{A}} \cdots e^{\mathcal{A}} a\left(f_{n}\right)^{*} e^{-\mathcal{A}}|0\rangle \\
& =a\left(e^{A} f_{1}\right)^{*} \cdots a\left(e^{A} f_{n}\right)^{*}|0\rangle \\
& =\left|e^{A} f_{1}, \ldots, e^{A} f_{n}\right\rangle .
\end{aligned}
$$

The existence of $C$ is a consequence of the spectral theorem. We call $\mathcal{C}$ the corresponding quadratic form in $a_{i}^{*}, a_{j}$. Through the repeated use of (A.12), one checks that applying $e^{\mathcal{A}} e^{\mathcal{B}} e^{\mathcal{A}}$ to the states (A.11) is the same as applying $e^{\mathfrak{E}}$. The semiboundedness of $A$ and $B$ ensures that the domain of their exponentials is the whole $L^{2}(V)$ and all quantities are well defined. Q.E.D.

\section{A.3 Proof of Lemma 4.2}

For any symmetric operator $A, \chi_{\Lambda} A \chi_{\Lambda} \leq \chi_{\Lambda}|A| \chi_{\Lambda}$. Hence $\left|\chi_{\Lambda} A \chi_{\Lambda}\right| \leq$ $\chi_{\Lambda}|A| \chi_{\Lambda}$ and $\operatorname{Tr}\left|\chi_{\Lambda} A \chi_{\Lambda}\right| \leq \operatorname{Tr}_{\Lambda}|A|$. When $A=D_{V}$, the convergence of the trace is proven by writing the further estimate $\operatorname{Tr}_{\Lambda}\left|D_{V}\right| \leq \operatorname{Tr}_{V}\left|D_{V}\right|$ and then summing an intregrable sequence of discrete eigenvalues. For $A=D$, one uses the Dirac-delta representation of the trace to find out that $\operatorname{Tr}_{\Lambda}|D|=|\Lambda|(2 \pi)^{-d} \int|\widehat{d}|$. The first assertion of the lemma has been proven.

As for the second part, let us write

$$
\begin{aligned}
& \operatorname{Tr}\left(\left|\chi_{\Lambda}\left(D_{V}-D\right) \chi_{\Lambda}\right|\right)=\operatorname{Tr}\left(U \chi_{\Lambda}\left(D_{V}-D\right) \chi_{\Lambda}\right) \\
= & \operatorname{Tr}_{V}\left(U \chi_{\Lambda} D_{V} \chi_{\Lambda}\right)-\operatorname{Tr}\left(U \chi_{\Lambda} D \chi_{\Lambda}\right)=T_{\ell}-T,
\end{aligned}
$$

where $U$ is the partial isometry $L^{2}(\Lambda) \longrightarrow L^{2}(\Lambda)$ that realizes the spectral decomposition as in [RS, Th. IV.10]. It is convenient to use the position representation for the bases. So, $\psi^{(k)}(x)=e^{i k \cdot x}$ and, as defined in Section 2 , $\psi_{V}^{(k)}=\psi^{(k)} \chi_{V}$. Let us work on $T_{\ell}$ : using the cyclicity of the trace one obtains

$$
\begin{aligned}
T_{\ell} & =\frac{1}{\ell^{d}} \sum_{k \in V^{\prime}}\left\langle\psi_{V}^{(k)}\left|\chi_{\Lambda} U \chi_{\Lambda} D_{V}\right| \psi_{V}^{(k)}\right\rangle \\
& =\frac{1}{\ell^{d}} \sum_{k \in V^{\prime}} \widehat{d}(k)\left\langle\psi_{V}^{(k)}\left|\chi_{\Lambda} U \chi_{\Lambda}\right| \psi_{V}^{(k)}\right\rangle \\
& =\frac{1}{\ell^{d}} \sum_{k \in V^{\prime}} \widehat{d}(k)\left\langle\psi^{(k)}\left|\chi_{\Lambda} U \chi_{\Lambda}\right| \psi^{(k)}\right\rangle,
\end{aligned}
$$


the last equality being due to the presence of the indicator functions $\chi_{\Lambda}$. In complete analogy with the above,

$$
T=\frac{1}{(2 \pi)^{d}} \int d k \widehat{d}(k)\left\langle\psi^{(k)}\left|\chi_{\Lambda} U \chi_{\Lambda}\right| \psi^{(k)}\right\rangle
$$

Since $\left|\left\langle\psi^{(k)}\left|\chi_{\Lambda} U \chi_{\Lambda}\right| \psi^{(k)}\right\rangle\right| \leq|\Lambda|$, it is obvious that (A.14) tends to (A.15) for $\ell \rightarrow \infty$.

$$
\text { Q.E.D. }
$$

\section{A.4 Proof of Lemma 5.1}

With regard to (4.9) and (4.12), $\operatorname{det}\left(1+\widetilde{\zeta} \chi_{\Lambda} D_{\varepsilon} \chi_{\Lambda}\right)$ is entire in $\widetilde{\zeta}$ (hence in $\zeta$ ) by [RS, Sec. XIII.17, Lemma 4(c)]. In order to evaluate its log (on the suitable Riemann surface) we need to avoid the zeros. Using [RS, Th. XIII.106], we want to make sure that $\sigma\left(-\widetilde{\zeta} \chi_{\Lambda} D_{\varepsilon} \chi_{\Lambda}\right)$ does not hit 1. Step 3 in Section 4 (see in particular formulas (5.22)-(5.23) and the last paragraphs) shows that this is never the case if $\widetilde{\zeta} \notin\left(-\infty,-z^{-1}-1\right]$, for $\mathrm{FD}$, or $\widetilde{\zeta} \notin\left[z^{-1}-1,+\infty\right)$, for BE.

Q.E.D.

Actually, we can say more. Consider FD, just to fix the ideas. We see from (5.22) that the "floating" endpoint of $\sigma\left(-\widetilde{\zeta} \chi_{\Lambda} D_{-} \chi_{\Lambda}\right)$ is strictly contained in the segment $\left(0,-\widetilde{\zeta} /\left(1+z^{-1}\right)\right)$, which means that $\widetilde{\zeta}$ is allowed to exceed slightly $G_{-}-1$, as given by (5.4), without any vanishing of (4.9). The above, and an analogous argument for BE, prove that for each finite $\Lambda$ the domain of analyticity of $\phi_{\varepsilon}^{\Lambda}(\zeta)$ is indeed strictly bigger than $G_{\varepsilon}$.

For the bosonic case this fact is related to Lemma 3.1. In a few words, $\chi_{\Lambda} D_{+} \chi_{\Lambda}$ can be thought of as defining a Hamiltonian $h_{\Lambda}^{\prime}$ in $L^{2}(\Lambda)$, via the relation

$$
\chi_{\Lambda} D_{+} \chi_{\Lambda}=\left(e^{-\beta h_{\Lambda}^{\prime}+\beta \mu 1_{\Lambda}}-1\right)^{-1} e^{-\beta h_{\Lambda}^{\prime}+\beta \mu 1_{\Lambda}} .
$$

(Compare this with the definition of $D_{V,+}$ given after formula (4.12)). Then all the calculations we have carried out in Section 5 are about the grand canonical ensemble of a system of bosons on $\Lambda$, with energy operator $h_{\Lambda}^{\prime}$ and chemical potential $\lambda+\mu$. The ground state of $h_{\Lambda}^{\prime}$ is strictly positive, in analogy to the Hamiltonian with Dirichlet b.c. In that case the upper bound for the chemical potential, $\lambda_{\max }(\Lambda)+\mu$, is stricly bigger than zero B, ZUK, $\mathrm{VLP}$. 


\section{A.5 The case $\Lambda=V$}

We discuss the less physical case of the large deviations for the density in the "large box" $V$.

Instead of first taking the limit $V \nearrow \mathbb{R}^{d}$ of the state $\langle\cdot\rangle_{\mu}^{V}$, and then looking at the asymptotic properties of the quantity $N_{\Lambda} /|\Lambda|$, for $\Lambda \nearrow \mathbb{R}^{d}$, we now consider the large deviations for the variable $N_{V} /|V|$ w.r.t. $\langle\cdot\rangle_{\mu}^{V}$, subject to the single limit $V \nearrow \mathbb{R}^{d}$. We call this the case $\Lambda=V$.

We immediately see that Theorem 3.2 is a trivial identity in this setup,

$$
\begin{aligned}
\lim _{V \nearrow \mathbb{R}^{d}} \frac{\log \left\langle e^{\beta \lambda N_{V}}\right\rangle_{\mu}^{V}}{\beta|V|} & =\lim _{V \nearrow \mathbb{R}^{d}} \frac{\log \Xi^{V}(\mu+\lambda)-\log \Xi^{V}(\mu)}{\beta|V|} \\
& =p(\mu+\lambda)-p(\mu)=g_{\mu}(\lambda) .
\end{aligned}
$$

Therefore the equivalent of Theorem 2.1 follows in the same way as outlined in Section 3 .

In the case $\Lambda=V$, however, it turns out that we can do more than just this. At least for some interesting cases, we can provide a lower bound for the large deviation relation even in the $\mathrm{BE}$ condensation regime, i.e. for $a \geq \rho_{c}$. This is how it is done.

With an eye to Section 3, we see that, due to (2.15), the problem is that there is no fixed $\lambda_{o}$ that verifies (3.22). In other words, no fixed value of the "extra" chemical potential $\lambda$ can be found such that the modified state

$$
\langle\cdot\rangle_{\lambda}=\frac{\left\langle\cdot e^{\beta \lambda N_{V}}\right\rangle_{\mu}^{V}}{\left\langle e^{\beta \lambda N_{V}}\right\rangle_{\mu}^{V}}=\langle\cdot\rangle_{\mu+\lambda}^{V}
$$

has an average density a (compare the above definition with (3.19)). On the other hand, as it is customary in the theory of Bose-Einstein condensation (e.g., $\mathrm{VLP}$ and references therein), one can take a variable $\lambda_{V}$ such that

$$
\frac{\left\langle N_{V}\right\rangle_{\lambda_{V}}}{|V|}=\rho^{V}\left(\mu+\lambda_{V}\right)=\frac{d}{d \mu} \frac{\log \Xi^{V}\left(\mu+\lambda_{V}\right)}{\beta|V|}=a .
$$

This formula is the analogue of (3.22). As regards the analogue of (3.23), it is not too hard to see that

$$
\frac{\beta}{|V|}\left[\left\langle N_{V}^{2}\right\rangle_{\lambda_{V}}-\left(\left\langle N_{V}\right\rangle_{\lambda_{V}}\right)^{2}\right]=\frac{d \rho^{V}}{d \mu}\left(\mu+\lambda_{V}\right) \approx|V| .
$$


That is, the variance of $N_{V} /|V|$ is of the order of a constant, at the limit. This means that our new state makes the density a average but not typical, in the sense that no law of large numbers holds. Thus, we cannot apply estimate (3.24) tout court.

However, we do not really need the density $a$ to be typical. The aforementioned argument goes through all the same provided that, for any $c<a$, we find an $\alpha \in(0,1)$ such that $\left\langle\chi_{|V|[a, c]}\left(N_{V}\right)\right\rangle_{\lambda_{V}} \geq \alpha$, for $V$ big enough.

This suggests that we look at the asymptotic distribution of $N_{V} /|V|$ in the grand canonical ensemble, when the infinite-volume on $V$ is taken under the restriction (A.19). This is a known object for several choices of $V, \epsilon(k)$ and $d$. For instance, when $V$ is a three-dimensional box (periodic, Neumann and Dirichlet b.c. apply) which expands isotropically and the energy is the non-relativistic $\epsilon(k)=k^{2} /(2 m)$, it is called the Kac distribution. Details of the computation can be found in [ZUK], whereas accounts or generalizations of the result are presented in many other works $[\mathrm{Q}, \mathrm{VLP}, \mathrm{VLZ}]$. It turns out that, for $a>\rho_{c}$,

$$
\lim _{V \nearrow \mathbb{R}^{d}}\left\langle\chi_{|V| J}\left(N_{V}\right)\right\rangle_{\lambda_{V}}=\frac{1}{a-\rho_{c}} \int_{J \cap\left[\rho_{c}, \infty\right)} \exp \left\{-\frac{x-\rho_{c}}{a-\rho_{c}}\right\} d x .
$$

Therefore the above claim holds true under certain assumptions and, in analogy with (3.25), we can write that, for $V \nearrow \mathbb{R}^{d}$,

$$
g\left(\lambda_{V}\right)-\lambda_{V} c+o(1) \leq \frac{\log \mathbb{P}\left(\left\{N_{V} \in|V| I\right\}\right)}{\beta|V|},
$$

with $\lambda_{V} \rightarrow-\mu_{-}$and $c$ arbitrarily close to $a$. Hence

$$
\liminf _{V \nearrow \mathbb{R}^{d}} \frac{\log \mathbb{P}\left(\left\{N_{V} \in|V| I\right\}\right)}{\beta|V|} \geq g(-\mu)+\mu a=f(a)=\sup _{x \in[a, b]} f(x) .
$$

We have thus shown that in the case $\Lambda=V$, the rate function for the large deviations in the density exists and is equal to the Legendre transform of the translated pressure, even in the $\mathrm{BE}$ condensation regime.

\section{References}

[B] R. BALIAN, From Microphysics to Macrophysics, Vols. I and II, Springer-Verlag, Berlin-Heidelberg, 1992. 
Large deviations for ideal quantum systems

[BR] O. Bratteli and D. W. Robinson, Operator Algebras and Quantum Statistical Mechanics, Vols. I and II, 2nd edition, Springer-Verlag, New York, 1997.

[C] J. T. Cannon, Infinite Volume of the Canonical Free Bose Gas States on the Weyl Algebra, Comm. Math. Phys. 29 (1973), 89-104.

[E] R. S. ElLIS, Entropy, Large Deviations, and Statistical Mechanics, Springer-Verlag, New York, 1985.

[F] M. E. Fisher, The Free Energy of a Macroscopic System, Arch. Rational Mech. Anal. 17 (1964), 377-410.

[FL] M. E. Fisher And J. L. Lebowitz, Asymptotic Free Energy of a System with Periodic Boundary Conditions, Comm. Math. Phys. 19 (1970), 251-272.

[G] H.-O. Georgir, Gibbs Measures and Phase Transitions, W. de Gruyter, Berlin-New York, 1988.

[Gi] J. Ginibre, Some Applications of Functional Integration in Statistical Mechanics, in: Statistical Mechanics and Quantum Field Theory, C. DeWitt, R. Stora, eds. Gordon and Breach, New York, 1971.

[H] K. Huang, Statistical Mechanics, J. Wiley \& Sons, New York, 1987.

[O] S. Olla, Large Deviations for Gibbs Random Fields, Probab. Theory Related Fields 77(3) (1988), 343-357.

[RS] M. Reed, B. Simon, Methods of Modern Mathematical Physics, Vols. I-IV, Academic Press, San Diego, 1972-1978.

[Ro] D. W. Robinson, The Thermodynamic Pressure in Quantum Statistical Mechanics, LNP 9, Springer-Verlag, New York, 1971.

[R] D. Ruelle, Statistical Mechanics: Rigorous Results, W. A. Benjamin, New York, 1969.

[SW] E. M. Stein And G. Weiss, Introduction to Fourier Analisys on Euclidean Spaces, Princeton University Press, Princeton, NJ, 1971. 
[T] C. J. Thompson, Mathematical Statistical Mechanics, Macmillan, New York, 1971.

[Ti] E. C. Titchmarsh, The Theory of Functions, 2nd edition reprinted, Oxford University Press, Oxford-New York, 1968.

[vLL] M. van Den Berg, J. T. Lewis and M. Lunn, On the General Theory of Bose-Einstein Condensation and the State of the Free Boson Gas, Helv. Phys. Acta 59 (1986), 1289-1310.

[vLP] M. van den Berg, J. T. Lewis And J. V. Pulè, A General Theory of Bose-Einstein Condensation, Helv. Phys. Acta 59 (1986), 1271-1288.

[vFS] A. C. D. van Enter, R. Fernández and A. D. Sokal, Regularity Properties and Pathologies of Position-Space RenormalizationGroup Transformations: Scope and Limitations of Gibbsian Theory, J. Stat. Phys. 72(5-6) (1993), 879-1167.

[Wa] G. N. Watson, A Treatise on the Theory of Bessel Functions, Cambridge University Press, Cambridge, 1922.

[W] J. Weidmann, Linear Operators in Hilbert Spaces, Springer-Verlag, New York, 1980.

[ZUK] R. M. Ziff, G. E. Uhlenbeck And M. KaC, The Ideal BoseEinstein Gas, Revisited, Phys. Rep. 32C (1977), 169-248. 